\title{
IMPACT OF BREAST RECONSTRUCTION ON MORTALITY AFTER BREAST CANCER: SURVIVAL ANALYSIS IN A COHORT OF 620 CONSECUTIVE PATIENTS
}

Bernardo Pinheiro de Senna Nogueira Batista', Suzana Sales de Aguiar ${ }^{1}$, Ana Carolina Padula Ribeiro-Pereira', Rosalina Jorge Koifman', Anke Bergmann¹

${ }^{1}$ National Cancer Institute - Rio de Janeiro (RJ), Brazil.

Background: Access to breast reconstruction is a complex and poorly understood aspect of survival. In the United States, although the rate of immediate reconstruction has tripled in the past 20 years, less than $40 \%$ of women undergoing a mastectomy will do so as part of the same procedure. Although there is common understanding that breast reconstruction is oncologically safe, published data on its impact on survival show conflicting and unjustified observations. Methods: We performed a secondary survival analysis in a fixed cohort of 620 consecutive patients who underwent mastectomy between August 2001 and November 2002 in a publicly financed tertiary cancer center. Results: Median followup was 118.4 months (6-172). Of the 620 patients, 253 (40.8\%) died during follow-up. And 94 (15.2\%) patients underwent breast reconstruction. An unadjusted Cox regression model with breast reconstruction as a time-dependent covariate showed a $60 \%$ reduction in the risk of death for patients who underwent reconstruction (crude HR=0.4; 95\%CI 0.25-0.65; $\mathrm{p}<0.001$ ). When adjusted for potential confounders registered in the primary study, the risk reduction was $44 \%$ (adjusted $\mathrm{HR}=0.56$; 95\%CI 0.34-0.92; $\mathrm{p}=0.02$ ). Conclusion: Access to breast reconstruction is associated with better survival after mastectomy. Although encouraging, these observations lack biological plausibility and inferences, suggesting that any causal effect is probably driven by confounding and/or interaction with unmeasured variables. The magnitude of the observed association, however, might suggest that, in settings where access to breast reconstruction is severely limited, patient selection for breast reconstruction could be an important drive of the observed association.

Keywords: Breast Neoplasm; Mammoplasty; Survival; Measures of Association; Confounding Factors. 